\title{
Constraints on the Parton Density Functions of the Proton by Measurements with the ATLAS Detector
}

\section{Mark Sutton*t}

The University of Sussex

E-mail: suttecern.ch

\begin{abstract}
Parton distribution functions (PDFs) are crucial ingredients for measurements at hadron colliders, since they describe the initial states and therefore critically impact the precision of cross section predictions for observables. This contribution will review recent precision analyses, where the PDFs play an important role and discuss the impact of several new ATLAS cross-section measurements on PDFs of the proton. Particular emphasis will be given to the determination of the strange and the gluon content of the proton.
\end{abstract}

The European Physical Society Conference on High Energy Physics

5-12 July, 2017

Venice

* Speaker.

${ }^{\dagger}$ On behalf of the ATLAS Collaboration 


\section{Introduction}

The LHC [1] has performed extremely well both during Run 1 - with 7 and $8 \mathrm{TeV}$ collisions - and, since 2015, during Run 2 with collisions of $13 \mathrm{TeV}$. This has allowed measurements of the $p p$ interaction cross sections over an unprecedentedly large kinematic region of many orders of magnitude in both the hard scale, and proton momentum fraction, $x$. The ATLAS detector [2] operated well during Run 1 and continues to perform well in Run 2, allowing an extensive and growing portfolio of precision measurements. These measurements can be used to provide precise constraints on the underlying theory of QCD and information of the partonic structure of the proton. This is essential for the study of all physics at the LHC, both for understanding the physics of the Standard Model, and physics beyond the Standard Model. In the region around the mass of the Higgs boson, constraints from some of the early LHC data have reduced the uncertainty on the parton distributions within the proton to a few percent, and at high masses - for instance in regions used for searches for contact interactions, or SUSY gluino searches - the uncertainty is still large.

Data from different physics processes provide constraints on the parton distribution functions (PDFs) which are fortunately sensitive to different contributions to the cross section: Data from deep inelastic scattering (DIS) at HERA are used to constrain the quark distributions. Jet production is sensitive to the gluon distribution and $\alpha_{s}$ already at tree level and heavy boson production is sensitive to the flavour composition of the quarks. By selecting specific flavours in the final states, either with or without accompanying jets, complimentary constraints on individual flavour distributions or the gluon can be obtained.

\section{ATLAS jet cross sections}

The ATLAS Collaboration has recently published measurements of the inclusive jet cross section in $p p$ collisions at $8 \mathrm{TeV}$ [3]. The inclusive jet cross section at $13 \mathrm{TeV}$ from LHC Run 2 has also recently been measured [4]. These show that in both cases the next-to-leading (NLO) calculation provides a reasonable qualitative descriptions of the cross section over the nine orders of magnitude spanned by the data. These cross sections are dominated by quark-gluon scattering for transverse momenta less than $1 \mathrm{TeV}$ and by the quark-quark scattering at high transverse momenta.

Figure 1 shows this data in more detail, showing the ratio of the theory over the measurement. The predictions include the uncertainty from the variation of each prediction when using the different fits from each PDF set $[5,6,7,8]$ and have been calculated using NLOJET++ [9]. The experimental uncertainties are typically smaller than the uncertainties on the theory. It is observed that the data in the individual pseudorapidity regions can generally be described well by the different PDFs except ABM11 [10] which is disfavoured. However when comparing all pseudorapidity regions simultaneously there is a tension.

Such comparisons are very dependent on the correlations of the systematic uncertainties, and as such ATLAS has tried many different correlation models for these uncertainties. Although some improvement is seen using such models, it is not sufficient to provide good agreement at NLO. It will be interesting to see to what extent this may also be the case when comparing with the recent predictions of the jet cross sections at next-to-next-to-leading order (NNLO) [11, 12]. 

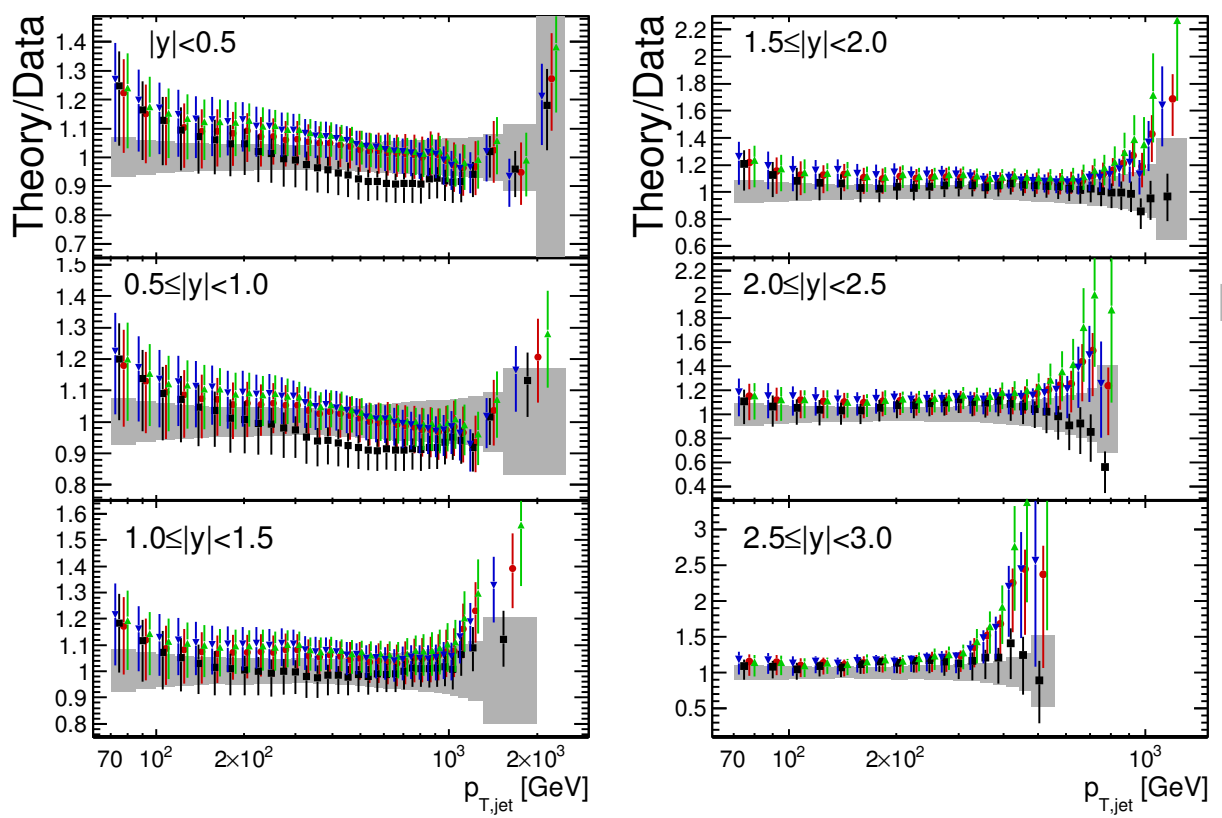

ATLAS

$L=20.2 \mathrm{fb}^{-1}$

$\sqrt{\mathrm{s}}=8 \mathrm{TeV}$

anti- $k_{t} R=0.4$

Data

NLO QCD

$\otimes k_{E W} \otimes k_{N P}^{\text {Pythia8 AU2CT10 }}$

$\mu_{\mathrm{R}}=\mu_{\mathrm{F}}=\mathrm{p}_{\mathrm{T}, \text { jet }}^{\max }$

CT14

HERAPDF2.0

+ NNPDF3.0

† MMHT2014

Figure 1: The ratio of the inclusive jet cross section NLO prediction to the data at $8 \mathrm{TeV}$ [3]. The data are shown as the grey band.

\section{Electroweak data}

The ATLAS Collaboration have also recently published data for Electroweak boson production with a precision analysis of inclusive $W$ and $Z$ production from the 2011 data, with the precise measurment of the $Z$ cross section both near the $Z$ pole, and in the low and high mass regions [13]. The NNLO corrections to these cross sections have been known for some time. Figure 2 shows the central $Z$ production cross section compared to the the NNLO prediction calculated with FEWZ $[14,15,16]$ using several PDF sets at NNLO, illustrating that in general NNLO calculation predicts slightly lower cross section with all PDF sets.

With the $W$ cross section, the lepton charge asymmetry cancels much of the gluon and sea quark contributions allowing a precise constraint on the valence $u$ and $d$ quark difference. Figure 2 also shows the asymmetry compared to the NNLO predictions with different PDF sets. Again there is a reasonably large spread of the prediction with respect to the very precise experimental measurements.

\section{QCD analysis}

Fits from PDF groups $[5,6,10]$ which include a constraint from neutrino data have typically resulted in a strange-to-down quark ratio of approximately 0.5. Fits to the early 2010 data [17] by the ATLAS Collaboration, denoted $e p W Z 12$, resulted in a PDF fit with an enhanced contribution to the strange quark density, with a ratio of around unity.

With the newer data [13], existing PDF sets can be profiled to determine which is the most consistent with the data and evaluate the approximate changes that might be expected to the sets if they had been performed including these data. Following this procedure for the MMHT [6] and 

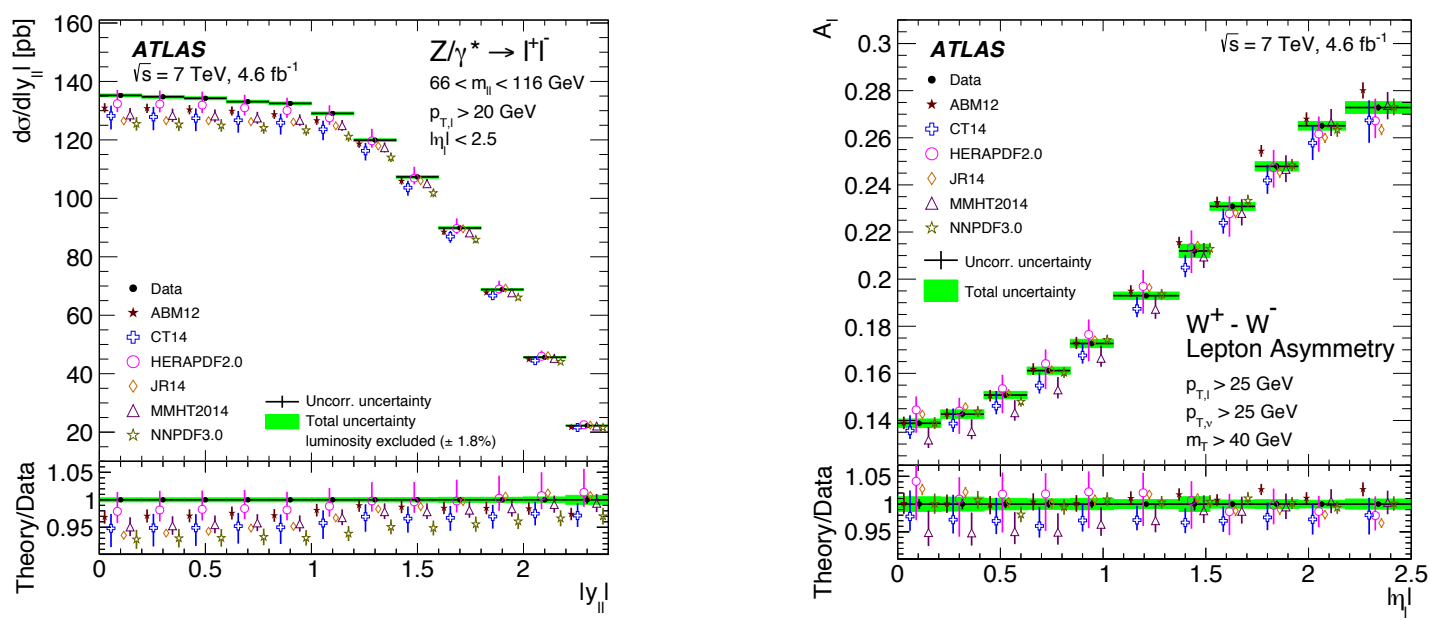

Figure 2: The $Z$ cross section from the 2011 ATLAS data (left) and the $W$ lepton charge asymmetry (right) from the 2011 ATLAS data [13].
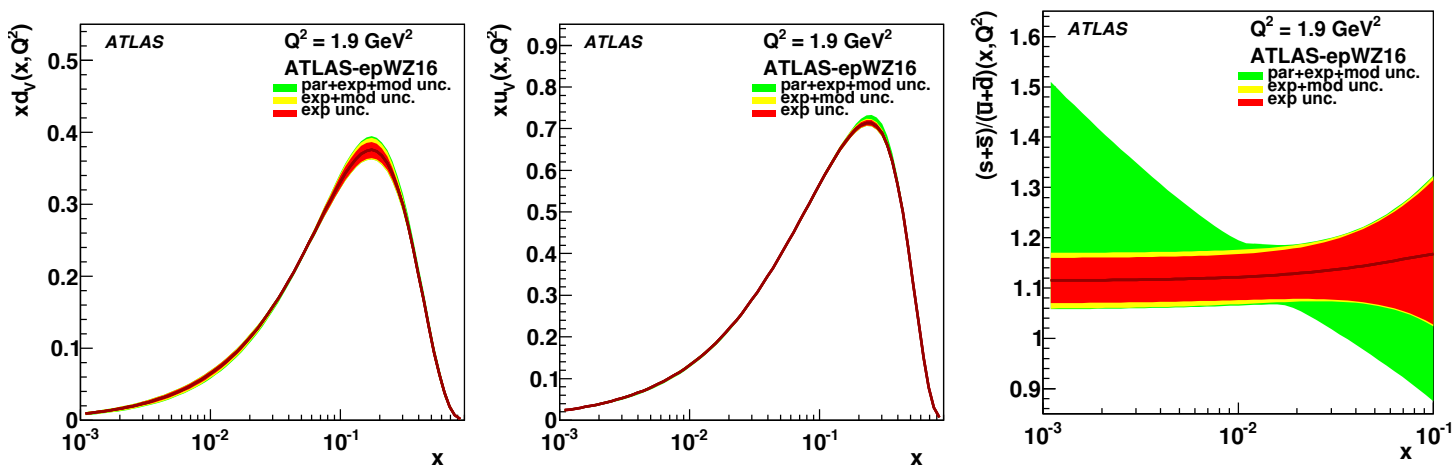

Figure 3: The $u$ and $d$ valence quark distributions, and the ratio of the strange quark density from the ATLAS epWZ16 QCD fit [13].

CT [18] sets, a reduction in the size of the uncertainty of the strange density is observed together with an increase from the values from the original fits, to around unity [13].

For a more rigorous evaluation, ATLAS has performed a new QCD analysis including this new data, with the usual 13 parameter fit, and with an alternative parameterisation where the parameters of the strange density are kept free to give a 15 parameter fit. With this new parameterisation a very precise determination of the $u$ and $d$ valence quark distributions is obtained with small uncertainties as shown in Figure 3. With this new fit to the new data, once again an enhanced strange contribution is observed consistent with the earlier observation, but with completely independent ATLAS data. This is shown in the ratio of strange to $u$ and $d$ sea quark distributions in Figure 3 .

During Run 2 the LHC has been operating with $p p$ collisions at $13 \mathrm{TeV}$ centre of mass energy. New measurements of the total inclusive electroweak boson cross sections and their ratios have been made [19]. The ratios are very precise as many of the experimental uncertainties cancel, and again a significant spread of the predictions from the different PDF sets are observed, while the data is still consistent with the epWZ12 fit with the enhanced strange.

The NNLO calculation of the $Z+$ jet cross section is available from several groups [20]. This 

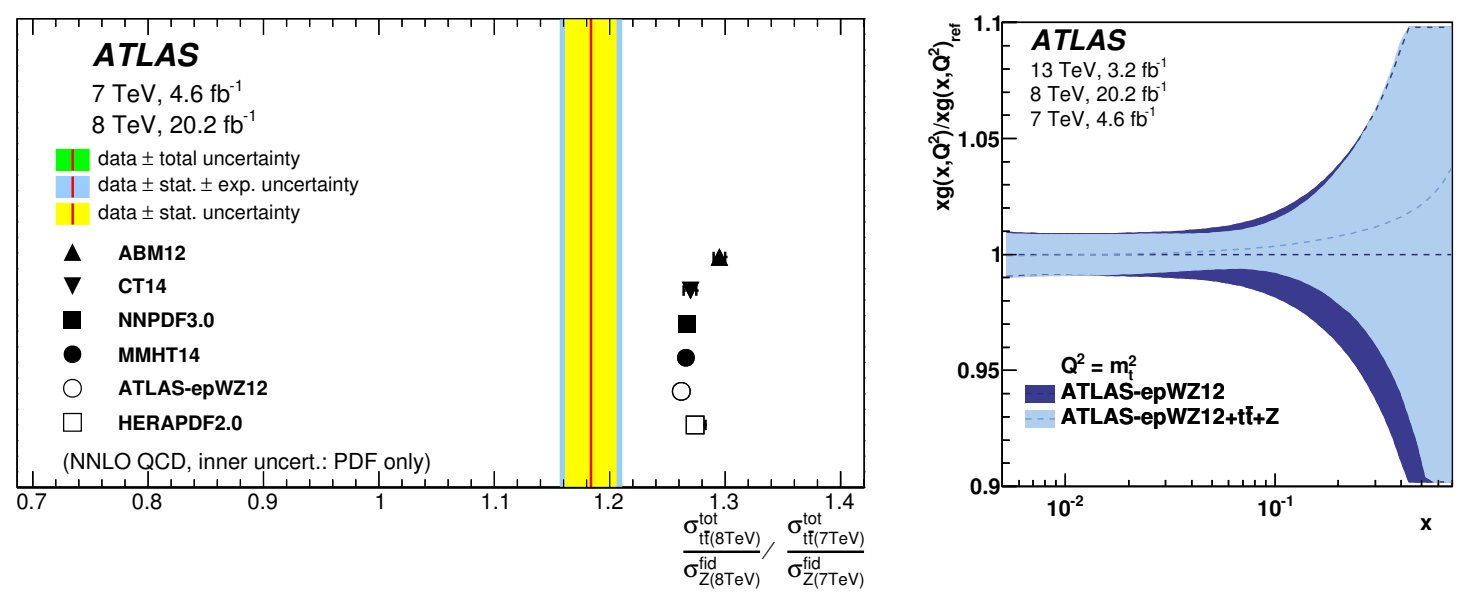

Figure 4: The Ratio of the $t \bar{t}$ to $Z$ cross sections at 7 and $8 \mathrm{TeV}$ collision energies (left) and the ratio of the gluon distributions profiled epWZ12 excluding or including the top and $Z$ data (right) [21].

will allow a complementary determination of the gluon distribution using the ATLAS data on $Z$ production in conjunction with a high transverse momentum jet.

\section{Heavy flavour, top, and prompt photon production}

The ATLAS Collaboration has a large number of cross section measurements involving top pair (ttbar) production. A new measurement [21] quantifies the ratios of the ttbar cross section with respect to the the $Z$ cross section at the different beam energies, 7, 8 and $13 \mathrm{TeV}$. Shown in Figure 4, a spread of the predictions is observed which suggests potential for a tighter constraint the parton distributions using these data. Most of the discrepancy between data and calculation in these ratios is arising predominantly from the $7 \mathrm{TeV}$ data. Once again profiling the PDF - this time using the epWZ12 fit - a significant reduction in the gluon uncertainty at large $x$ is observed as also seen in Figure 4. A moderate reduction in the light quark sea for intermediate $x$ values is also observed with the constraint on the sea coming largely from the $Z$ data, as would be expected.

New measurements of inclusive prompt photon production [22] are also available. For this process the cross section is dominated by the $u$-gluon interactions at tree level. For these new data once again the theoretical uncertainties at NLO, this time from JETPHOX [23, 24], are larger than the experimental uncertainties. The prompt photon cross section is now also available at NNLO [25].

\section{Summary}

The ATLAS Collaboration has an extensive and growing portfolio of precision measurements that are useful for constraining the proton PDF, although only a small selection could be discussed here. Notable results that space did not allow to be discussed include the measurement of $Z+b$ production, and $W+$ charm production. These have previously allowed a complementary and more direct extraction of the strange quark density itself consistent with the enhanced strange density extraction described here. There remains a large, untapped potential to extract important additional 
measurements from the Run 1 data, but also a large and growing reservoir of $13 \mathrm{TeV}$ collision data. Together with the many recent developments in the NNLO calculations that are required to fully exploit these data, the prospects appear very good.

A great deal of progress has been made in the exploitation of the LHC data, however, this is really only the beginning of the journey towards realising the full potential of the precise data now becoming available, so there will be extremely interesting times ahead.

\section{References}

[1] L. Evans and P. Bryant, LHC Machine, Tech. Rep. JINST 3:S08001 (2008), DOI:10.1088/1748-0221/3/08/S08001.

[2] ATLAS Collaboration; JINST 3:S08003 (2008), DOI:10.1088/1748-0221/3/08/S08003.

[3] ATLAS Collaboration; JHEP 09 (2017) 020, DOI:10.1007/JHEP09(2017)020.

[4] ATLAS Collaboration; CERN-EP-2017-157, hep-ex/1711.02692.

[5] S. Dulat, et al; Phys. Rev. D 93 (2016) 033006, [hep-ph/1506.07443], DOI:10.1103/PhysRevD.93.033006.

[6] L. Harland-Lang, A. Martin, P. Motylinski, and R. Thorne; Eur. Phys. J. C 75 (2015) 204, [hep-ph/1412.3989], DOI:10.1140/epjc/s10052-015-3397-6.

[7] NNPDF Collaboration; JHEP 04 (2015) 040, [hep-ph/1410.8849] DOI:10.1007/JHEP04(2015)040.

[8] H1 and ZEUS Collaborations; Eur. Phys. J. C75, 580 (2015), [hep-ph/1511.05402], DOI:10.1140/epjc/s10052-015-3710-4.

[9] Z. Nagy; Phys. Rev. D 68 (2003) 094002, [hep-ph/0307268] DOI:10.1103/PhysRevD.68.094002.

[10] S. Alekhin, J. Bluemlein, and S.-O. Moch; DESY 13-020, [hep-ph/1302.1516].

[11] J. Currie, E. W. N. Glover and J. Pires; Phys. Rev. Lett. 118072002 (2017), DOI:10.1103/PhysRevLett.118.072002.

[12] J. Currie et al; Phys. Rev. Lett. 119152001 (2017), DOI:10.1103/PhysRevLett.119.152001.

[13] ATLAS Collaboration; Eur. Phys. J. C 77 (2017) 367, DOI:10.1140/epjc/s10052-017-4911-9.

[14] R. Gavin, Y. Li, F. Petriello and S. Quackenbush; Comput. Phys. Commun. 182 (2011) 2388, [hep-ph/1011.3540], DOI:10.1016/j.cpc.2011.06.008.

[15] R. Gavin, Y. Li, F. Petriello and S. Quackenbush; Comput. Phys. Commun. 184 (2013) 209, [hep-ph/1201.5896], DOI:10.1016/j.cpc.2012.09.005.

[16] Y. Li and F. Petriello, Phys. Rev. D 86 (2012) 094034, [hep-ph/1208.5967], DOI:10.1103/PhysRevD.86.094034.

[17] ATLAS Collaboration; Phys. Rev. Lett. 109 (2012) 012001, DOI:10.1103/PhysRevLett.109.012001.

[18] S. Dulat et al; Phys. Rev. D 93 (2016) 033006 [hep-ph/1506.07443], DOI:10.1103/PhysRevD.93.033006.

[19] ATLAS Collaboration; Phys. Lett. B 759 (2016) 601, DOI:10.1016/j.physletb.2016.06.023.

[20] A. Gehrmann-De Ridder et al; Phys. Rev. Lett. 117 (2016) no.2, 022001, DOI:10.1103/PhysRevLett.117.022001. 
[21] ATLAS Collaboration; JHEP 02 (2017) 117, DOI:10.1007/JHEP02(2017)117.

[22] ATLAS Collaboration; Phys. Lett. B 770 (2017) 473, DOI:10.1016/j.physletb.2017.04.072.

[23] P. Aurenche, M. Fontannaz, J. Ph. Guillet, E. Pilon and M. Werlen, Phys. Rev. D 73 (2006) 094007, and references therein, [hep-ph/0602133], DOI:10.1103/PhysRevD.73.094007.

[24] S. Catani, M. Fontannaz, J. Ph. Guillet and E. Pilon; JHEP 0205 (2002) 028, [hep-ph/0204023], DOI:10.1088/1126-6708/2002/05/028.

[25] J. M. Campbell, R. K. Ellis and C. Williams; Phys. Rev. Lett. 118, 222001 (2017), DOI:10.1103/PhysRevLett.118.222001. 